Relations industrielles

Industrial Relations

\title{
Traité de droit administratif canadien et québécois, par René Dussault, tomes I et II, Québec, P.U.L., 1974, 2016 pp.
}

\section{Rodrigue Blouin}

Volume 30, numéro 2, 1975

URI : https://id.erudit.org/iderudit/028617ar

DOI : https://doi.org/10.7202/028617ar

Aller au sommaire du numéro

Éditeur(s)

Département des relations industrielles de l'Université Laval

ISSN

0034-379X (imprimé)

1703-8138 (numérique)

Découvrir la revue

Citer ce compte rendu

Blouin, R. (1975). Compte rendu de [Traité de droit administratif canadien et québécois, par René Dussault, tomes I et II, Québec, P.U.L., 1974, 2016 pp.]

Relations industrielles / Industrial Relations, 30(2), 274-276.

https://doi.org/10.7202/028617ar

Tous droits réservés @ C Département des relations industrielles de l'Université Laval, 1975
Ce document est protégé par la loi sur le droit d'auteur. L'utilisation des services d'Érudit (y compris la reproduction) est assujettie à sa politique d'utilisation que vous pouvez consulter en ligne.

https://apropos.erudit.org/fr/usagers/politique-dutilisation/ 
renseignements généraux. On devra cependant fouiller ailleurs pour trouver des études plus approfondies.

\section{Université Laval}

Jean SEXTON

Organizational Behavior: Critical Incidents and Analysis, par John F. Murray et Thomas J. Von der Embse, Columbus, Ohio, Charles E. Merrill Publishing Compay, 1973, 304 pp.

Comme le titre l'indique, ce volume est un recueil d'incidents critiques couvrant les aspects les plus importants de la dimension humaine des organisations de travail. L'introduction présente l'incident critique comme un instrument d'apprentissage, différent de la méthode des cas en administration. De fait, l'incident critique cherche plutôt à développer chez l'étudiant une habileté à identifier et à analyser une situation, sans procéder à la recherche de solutions possibles, puisque la situation à analyser est décrite en utilisant un minimum d'information. Cet instrument pédagogique est conçu de manière à permettre à l'étudiant de reconnaître des concepts et des théories déjà exposés par un professeur ou dans des textes choisis. Il vise donc à développer chez l'étudiant la compréhension des concepts et des résultats de recherche en les applicant à l'étude de situations très particulières. Il fait aussi largement appel à la faculté d'imagination chez l'étudiant qui doit indiquer la nature de l'information additionnelle qui serait nécessaire à une analyse plus poussée de la situation.

La deuxième partie du recueil comprend trente incidents distribués également sous les thèmes suivants: la perception, la motivation, la communication, les statuts et les rôles, le conflit, l'autorité, le pouvoir, le leadership, le changement et le climat organisationnel. Chaque situation est suivie d'une analyse que l'étudiant peut utiliser pour vérifier dans quelle mesure il a réussi à reconnaître et à appliquer les éléments conceptuels et théoriques que les auteurs voulaient mettre en évidence dans la description de la situation. La troisième partie est une présentation «en vrac» de situations non-regroupées sous un thème particulier, mais couvrant une ou plusieurs dimensions des sciences du comportement appliquées à l'étude des organisations.

Il m'arrive assez souvent d'utiliser cet instrument au cours de mon enseignement. Pour les étudiants qui ont déjà une expérience des organisations de travail, cet instrument s'avère rentable au plan pédagogique, puisqu'ils ont plus de facilité que les étudiants réguliers à reconstituer les éléments d'une situation et à identifier les compléments d'information qu'il faut apporter. Les étudiants réguliers qui possèdent des connaissances plutôt sommaires de la structure et $\mathrm{du}$ fonctionnement des organisations se trouvent « désemparés 》, démunis devant la pénurie d'informations. Ceci devient pour eux une excuse pour ne pas fournir l'effort qu'on attend d'eux. Il s'agit alors d'utiliser cet instrument pour introduire le sujet qu'on veut traiter; ou encore, illustrer un thème que l'on vient de développer devant eux. Ce sont là, à mon avis, les limites que comporte l'utilisation de cet ouvrage.

Laurent BÉLANGER

\section{Université Laval}

Traité de droit administratif canadien et québécois, par René Dussault, tomes I et II, Québec, P.U.L., 1974, 2016 pp.

Ce traité constitue une magistrale synthèse du droit administratif. L'auteur y étudie, par une approche intégrée et ordonnée, la plupart des problèmes juridiques qui se rapportent à l'administration publique. Le lecteur sentira tout au long de l'exposé un indéniable souci de réaliser un équilibre entre les objectifs de la recherche scientifique et ceux de la pédagogie.

Le plan de l'ouvrage est classique et de consultation facile. Trois parties, dont deux font l'objet du tome premier, regroupent successivement les matières concernant l'organisation, les pouvoirs et le contrôle de l'administration. Ces trois parties sont précédées d'un chapitre préliminaire de type introductif. La. lecture de l'ouvrage est rendue agréable par un style alerte et concis. L'auteur fait un usage constant de références infra-paginales. De nombreuses et intéressantes citations en langue française et anglaise parsèment le texte. L'utilisation des références au bas de page faite par l'auteur 
pour rapporter des citations additionnelles ou faire des propos complémentaires est bien pensée. Les problèmes de fond donnent lieu à des analyses complètes et bien présentées. Elles sont généralement suivies d'une synthèse où l'auteur apporte quelques observations générales complémentaires, critiques et recommandations.

Le chapitre préliminaire est consacré à situer d'abord la place de l'administration en régime démocratique puis à préciser la notion et le champ d'application du droit administratif et ses sources juridiques. Dans ses propos consacrés au rattachement de notre droit administratif au droit anglo-américain, l'auteur souligne qu' "il apparaît incorrect ou $d u$ moins très peu sûr d'affirmer dans l'absolu, comme l'ont fait certains auteurs, que les règles du droit anglais ou britannique relatives au droit administratif ne s'appliquent pas au Québec» (p 17). «Quoi qu'il en soit, sur le plan pratique, on ne saurait faire l'étude du droit administratif au Québec et au Canada, sans se référer de façon constante aux décisions des tribunaux britanniques sur la question» (p. 22). On regrette que l'auteur n'indique pas quelle valeur il entend attribuer aux décisions américaines. On peut enfin imaginer que l'importance donnée à la jurisprudence britannique est susceptible de donner cours à une belle polémique.

Trois thèmes principaux sont étudiés dans la première partie de l'ouvrage consacrée à l'organisation de l'administration : les structures, le personnel et les biens. L'auteur consacre quelques propos préliminaires pour expliquer son affirmation à l'effet que «le terme administration désigne l'ensemble des organes administratifs fédéraux et québécois, qu'ils soient centralisés, de façon concentrée ou déconcentrée, ou décentralisés sur le plan fonctionnel ou territorial» (p. 34). Le chapitre consacré aux structures administratives présente successivement les structures centrales puis les structures non-centrales. Aucun effort n'est ménagé pour donner la vision la plus complète possible et la plus intelligible à celui qui veut saisir adéquatement l'ensemble complexe de la réalité administrative par laquelle l'administration accomplit la grande variété des tâches et fonctions qui lui échoie. L'étude du personnel de l'administration, au se- cond chapitre, est faite en deux temps : analyse de la relation administrationfonction publique, puis étude du régime juridique de la fonction publique. Ici aussi les matières sont bien traitées bien que l'on ait pu s'attendre à plus d'informations sur le régime des sanctions et protections juridictionnelles. Pour ceux qui s'intéressent aux relations du travail, on remarquera cette importante prise de position : "Si l'esprit du service public commande que le statut juridique de la fonction publique situe l'administration dans une relation d'autorité par rapport aux fonctionnaires, il est par ailleurs conforme à l'optique moderne du droit du travail que cette situation juridique fasse place à la négociation collective» (pp. 444-445). Le troisième chapitre, consacré aux biens de l'administration, présente successivement la notion du domaine public (ensemble des biens et propriétés de l'Etat), la répartition, l'exploitation et la protection de ce domaine. L'épineux problème des droits des Indiens (pp. 577 à 582) est traité dans les propos sur l'exploitation et non pas, comme on aurait pu s'y attendre, dans la discussion sur la répartition du domaine public. Plusieurs seront sans doute intéressés à se pencher de façon approfondie sur l'observation suivante : «il apparaît souhaitable d'abolir le principe juridique qui veut que la propriété des lits et rives des cours d'eau non navigables et non flottables soit reconnue aux propriétaires riverains» (p. 622). «D'une manière générale il convient de reconnaître une nouvelle limite à la propriété privée, qui est extérieure à la loi ou aux règlements. Cette limite tient essentiellement au rôle social que doit jouer la propriété»(p. 622).

La deuxième partie de l'ouvrage présente sous trois thèmes les principaux problèmes concernant les pouvoirs de l'administration: notion générale des pouvoirs, puis pouvoir réglementaire pour en arriver au pouvoir contractuel. Le premier chapitre donne un aperçu de la nature et du régime des pouvoirs de l'administration. Le terme pouvoir y est pris exclusivement dans le sens de fonction ( on parle alors des fonctions de toute nature exercées par un organe, notamment par l'administration, dans le cours de son action» p. 669). L'étude faite est très succinte. Le pouvoir réglementaire, sa nature et ses limites, forme la matière du second chapitre. L'analyse 
des limitations de ce pouvoir est particulièrement bien rodée. Les praticiens notamment y trouveront une source inestimable d'informations. On notera que l'auteur semble préconiser «l'instauration d'un contrôle parlementaire étroit sur l'exercice du pouvoir réglementaire» (p. 864). Cette suggestion pourrait faire l'objet d'une recherche fort intéressante. Le troisième et dernier chapitre de cette partie analyse le pouvoir contractuel de l'administration. Elaboration, conclusion et exécution du contrat administratif sont étudiées en trois mouvements. Des propos introductifs précisent la notion de contrat administratif et expliquent le droit du contrat administratif. "On se rend compte que si, au départ, on se refuse à appliquer un droit spécial aux contrats de l'administration, le particularisme poussé de ces contrats a malgré tout entraîné l'élaboration de règles dérogatoires, à bien des points de vue, au droit commun» (p. 880). Les règles dégagées sur le pouvoir contractuel confirme bien cette mise-en-contexte. On trouve dans ce chapitre d'intéressants développements bien que succints.

Dans la troisième partie, l'auteur analyse successivement les moyens de pourvoi en droit administratif, le contentieux de la légalité administrative et celle de la responsabilité. Le premier chapitre est consacré en majeure partie au pouvoir judiciaire. Une analyse particulièrement succinte est présentée sur les pouvoirs administratif et parlementaire. A la lecture de ce chapitre, on ne peut avec l'auteur que demeurer «perplexe devant la complexité et la multitude des recours qui s'offrent aux administrés insatisfaits des actes et décisions de l'administration » (p. 1067). Le contentieux de la légalité administrative, au second chapitre, élabore successivement sur l'existence et l'exercice du pouvoir de contrôle judiciaire. Il s'agit en fait d'une mise-à-jour de l'ouvrage publié par Me Dussault en 1969, Le contrôle de l'administration judiciaire au Québec, P.U.L. On peut regretter que l'auteur n'ait pas élaboré sur la jurisprudence et les opinions doctrinales récentes qui tendent à affirmer que l'action directe en nullité n'existe plus lorsqu'il s'agit de l'exercice par la Cour supérieure de son pouvoir de contrôle sur les procédures et décisions des tribunaux inférieurs. Quoi qu'il en soit, la mise-à-jour reste excellente. On notera que l'auteur préconise d'en arriver «par une formulation différente des clauses privatives et par l'adoption de nouvelles dispositions appropriées, à élaborer un corps de règles ou de principes juridiques indépendants de la notion de l'ultra vires qui leur permettraient d'intervenir et de contrôler l'activité de l'administration (...)» (p. 1423). Le contentieux de la responsabilité administrative fait l'objet du dernier chapitre. La responsabilité délictuelle ou quasi-délictuelle est d'abord présentée et elle donne lieu à d'intéressants développernents de droit comparé. Une section est ensuite consacrée à la responsabilité contractuelle mais elle est succinte. Grâce à ce chapitre, «on se rend compte que le droit de la responsabilité coniractuelle de l'administration, comme celui de sa responsabilité délictuelle d'ailleurs, diffère à maints égards du droit commun, dans la mesure où il doit répondre à des besoins nettement particuliers 》 ( $\mathrm{p}$. 1538).

Dans la conclusion générale, Me Dussault se penche sur les moyens de pourvoi dont on peut doter les citoyens face à l'administration. L'auteur fait un ensemble de recommandations sur les moyens de pourvoi judiciaire et parlementaire. L'attention du lecteur sera notamment attirée par l'idée mise-en-place «d'un véritable régime d'aide juridique » face à l'administration (p. 1570).

L'ouvrage est complété par une bibliographie, une table de jurisprudence, une table des lois, un index des auteurs et des juges et, enfin, par un index analytique. Ces instruments de travail sont très bien confectionnés et d'une rare richesse.

On aura pu le constater, ce Traité de droit administratif canadien et québécois de Me Dussault est une oeuvre colossale. L'ouvrage sera sans nul doute un point tournant dans l'enseignement et la pratique du droit administratif. Il marquera, en quelque sorte, une date importante pour l'avancement de la science juridique. On ne peut que conclure que ce traité doit être considéré comme un instrument de travail indispensable et privilégié pour les étudiants, chercheurs et praticiens du droit et de l'administration publique.

Rodrigue BLOUIN

Université Laval 